\title{
The Role of Echocardiography in Heart Failure
}

\author{
Thomas H. Marwick \\ Menzies Institute for Medical Research, Hobart, Tasmania, Australia
}

\begin{abstract}
Data from echocardiography provide a cornerstone in the management of heart failure. All imaging techniques can provide an ejection fraction, but the versatility of echocardiography makes it unique in the provision of volumes, diastolic function, right ventricular function, hemodynamics, and valvular regurgitation. The early detection of heart failure has been facilitated by the assessment of global longitudinal strain, which is also useful in later heart failure for the assessment of left ventricular synchrony. The use of echocardiography has been associated with favorable outcomes, probably on the basis of facilitation of appropriate therapy. This review examines the evidence provided by echocardiography and its application in specific settings. Although the guidelines emphasize that no single test satisfies all imaging requirements in heart failure, and other modalities can provide additional information about specific questions (especially tissue characterization), echocardiography is indispensable in the management of heart failure.
\end{abstract}

Key Words: 2D echocardiography; 3D echocardiography; heart failure; strain

J Nucl Med 2015; 56:31S-38S

DOI: $10.2967 /$ jnumed.114.150433

\section{$\mathbf{P}$}

atients with heart failure (HF) receive medical care from a variety of providers and at a variety of disease stages. Because the cardiology literature often focuses on severe or inpatient HF, including devices, there is a risk of neglecting diagnosis and management in the large number of ambulatory HF patients in the community. The availability, relatively low cost, and versatility of echocardiography make it the imaging procedure of choice in these patients. Even in the hospital, the ability to perform echocardiography during procedures and at the bedside, including the intensive care environment, makes this test indispensable (1).

Echocardiographic imaging has several targets in the HF population, including the assessment of myocardial structure and function, valvular disease, and hemodynamics (Table 1). In addition, ultrasound techniques can provide information on abnormal myocardium (based on strain or integrated backscatter abnormalities), although the ability of ultrasound to characterize tissue (scar vs. inflammation or infiltration) is far inferior to that of cardiac MR. Of course, ultrasound evaluation should not occur in isolation, being incremental to clinical and hemodynamic assessment, sophisticated imaging such as cardiac MR, and genetic analysis (2).

Received Apr. 2, 2015; revision accepted Apr. 30, 2015.

For correspondence or reprints contact: Thomas H. Marwick, Menzies Institute for Medical Research, Private Bag 23, Hobart, Tasmania 7000, Australia.

E-mail: tom.marwick@utas.edu.au

COPYRIGHT (C) 2015 by the Society of Nuclear Medicine and Molecular Imaging, Inc.

\section{ECHOCARDIOGRAPHIC TOOLS FOR HF ASSESSMENT}

All modalities of echocardiography are useful in the assessment of the HF patient. Historically, this process began with M-mode echocardiography, a technique that remains helpful for accurate assessment of wall and chamber dimensions. The use of 2-dimensional (2D) echocardiography has added the ability to assess left ventricular (LV) volume and valvular disease. The progression to 3-dimensional (3D) echocardiography has enhanced the accuracy and reliability with which chamber volume and function are measured, as well as the mechanistic evaluation of regurgitation. Doppler assessment of hemodynamics has become an indispensable adjunct to HF evaluation, including assessment of pulmonary artery and right atrial pressure, LV filling, and regurgitant valve lesions (3). The recent development of myocardial strain has facilitated the assessment of long-axis function, which is sensitive to the preclinical phases of HF, as well as the evaluation of LV synchrony (4). Finally, the bedside application of handheld ultrasound to the assessment of intravascular volume and pulmonary congestion appears to be a feasible alternative to clinical examination, in view of the attenuation of clinical examination skills (5).

\section{Ejection Fraction (EF)}

Visual estimation of EF is widely used, and for many years it has been known that the eye of an experienced observer is comparable to the performance of a trackball (6). However, this approach may be misleading when the rhythm is irregular (requiring capture of multiple cardiac cycles), when the LV size is very large or very small, and when the extremes of heart rate are present. There are numerous quantitative approaches with echocardiography. Fractional shortening and the Teicholtz method for estimation of EF from M-mode imaging have been superseded by measurements of LV volumes in apical views using the biplane method of Simpson (7). Nonetheless, even with 2D imaging, the likelihood that repeated images will cut through the same 3D plane is small, and therefore repeated testing has a high likelihood of producing variable volume and $\mathrm{EF}$ results. When $\mathrm{EF}$ is close to the criteria assigned for electrical device insertion, variation may have important implications (Fig. 1). The use of 3D echocardiography provides analogous EF results (8) but has the advantage of less test-retest variation (9).

$\mathrm{EF}$ has a central role in the management of HF, identifying patients who are likely to respond to therapy for HF with reduced $\mathrm{EF}$ as well as patients who will benefit from device therapy, either implanted defibrillators or cardiac resynchronization. Indeed, the reason for outcome benefit in patients undergoing echocardiography at the time of HF presentation is likely connected to the importance of EF in selecting prognostically beneficial therapy $(10,11)$. Although the limitations of this technique related to geometric assumptions may be avoided by 3D imaging (Fig. 2), EF measures by either method are susceptible to inaccurate tracing, load dependence, poor test-retest reliability, and limited prognostic value when the measure is close to the reference range (3). 
TABLE 1

Measurements, Indications, and Modalities Used in Echocardiographic Evaluation of HF

\begin{tabular}{|c|c|c|}
\hline Measurement & Indication & Modality \\
\hline $\mathrm{EF}$ & $\begin{array}{l}\text { Classification, risk assessment; } \\
\text { selection for implantable cardioverter } \\
\text { defibrillator and cardiac } \\
\text { resynchronization therapy }\end{array}$ & $2 \mathrm{D}$ or $3 \mathrm{D}$ echocardiography, contrast \\
\hline Non-EF indices of function & Risk assessment & Doppler $\mathrm{dP} / \mathrm{dt}$ \\
\hline Mitral regurgitant orifice area & Quantification of mitral regurgitation & Color and CW Doppler \\
\hline Transmitral and annular velocities & $\begin{array}{l}\text { Assessment of diastolic function and } \\
\text { filling pressure }\end{array}$ & $\begin{array}{l}\text { Pulsed-wave tissue and blood-flow } \\
\text { Doppler }\end{array}$ \\
\hline Left atrial volume & Prognosis, diastolic evaluation & $2 \mathrm{D}$ or $3 \mathrm{D}$ echocardiography \\
\hline RV assessment & Prognosis, evaluation for assist device & $\begin{array}{l}\text { RV strain, tissue Doppler, tricuspid } \\
\text { annular plane systolic excursion, } \\
\text { fractional area change, etc. }\end{array}$ \\
\hline Regional function & Assessment of CAD & Wall motion analysis, strain \\
\hline Regional timing & Site of greatest maximal delay & $\begin{array}{l}\text { Cardiac resynchronization therapy } \\
\text { selection }\end{array}$ \\
\hline
\end{tabular}

Strain is a means of measuring myocardial deformation, which has been measurable clinically for more than $15 \mathrm{y}$. The initial methodology involved tissue Doppler (which was angle-dependent) but speckle tracking of gray-scale images has been the method of choice for over a decade (12). Global longitudinal strain is a robust measure of LV long-axis function that is particularly useful for assessing the preclinical phases of HF (Fig. 3). Strain rate is better than strain as a marker of contractility, although the temporal resolution of speckle tracking at normal frame rates makes it more difficult to measure accurately than with tissue Doppler-based approaches. Regional strain has been

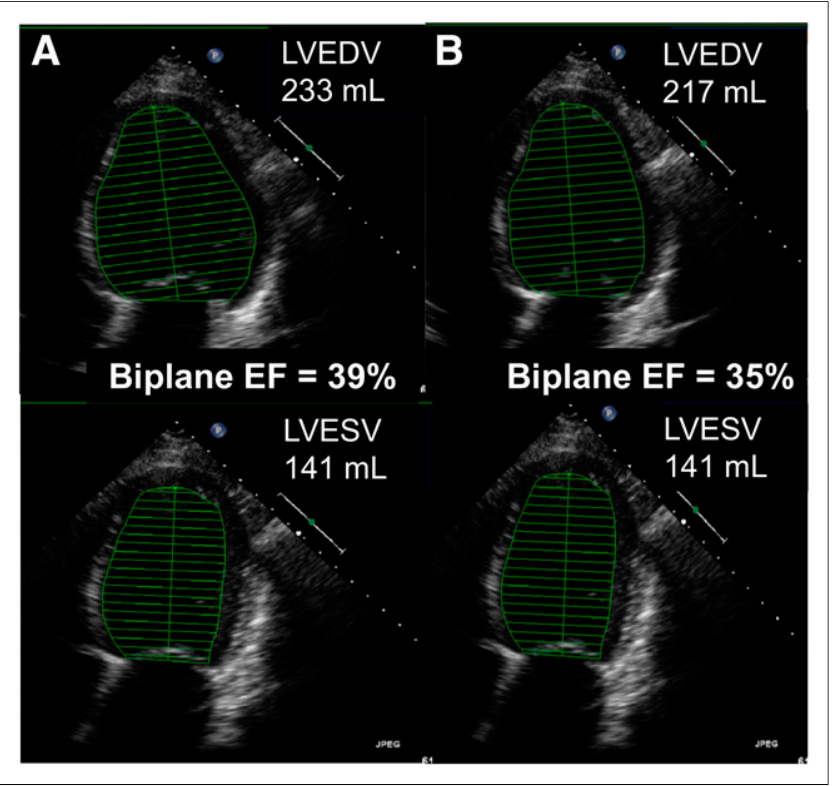

FIGURE 1. Calculation of LV volumes and EF from biplane Simpson's formula using $2 \mathrm{D}$ echocardiography. Repeated measurement at same examination (A vs. B) shows that relatively minor $(7 \%)$ difference in volume calculation translates to EF change sufficient to change a decision. LVEDV = LV end-diastolic volume; LVESV = LV end-systolic volume. validated against measurements with cardiac MR and microcrystals but lacks the spatial averaging of global longitudinal strain.

\section{Load-Independent Parameters}

A major goal of echocardiographic assessment in $\mathrm{HF}$ is evaluation of the performance of the left ventricle, which is dependent on not only contractile state but also load. The possible ways to address this issue include assessment of afterload and preload in order to correct standard measures (e.g., EF) or measurement of contractility independent of loading. However, many investigators consider the rationale for defining a load-independent parameter to be inherently flawed because cardiac function per se is linked to loading mechanics.

Measurement of Load. Afterload is reflected by systolic wall stress, which has conventionally been approximated by the product of force (blood pressure) and LV size (expressed as LV internal dimension) and is inversely proportionate to wall thickness, as derived from the law of Laplace (12). Unfortunately, there are several problems with this approach. First, the geometric simplifications are excessive. Second, peak wall stress occurs in early systole. Third, abnormal arterial properties (including abnormalities of the aortic reservoir and wave reflection) may cause LV systolic pressure to be overestimated from cuff systolic pressure. For these reasons, wall stress (13) is a difficult parameter to apply clinically, and consequently, blood pressure is often used as the marker of afterload. Likewise, preload assessment is difficult, although LV diastolic pressure can be estimated by echocardiography.

Load-Corrected Parameters. Essentially, all clinical parameters of LV function are load-dependent. However, some of the more egregious sources of inaccuracy can be avoided. LV midwall shortening is less dependent on LV geometry than are endocardial measurements. Although more recently replaced by LV strain measurements, LV midwall shortening compensates for LV hypertrophy rather than afterload. The association between loading and inotropic state is conventionally expressed by pressure-volume loops at different workloads and by contractility measured from end-systolic elastance. Noninvasive approaches to approximating this association have focused on the pressure-volume relationship and have been facilitated by $3 \mathrm{D}$ imaging. 


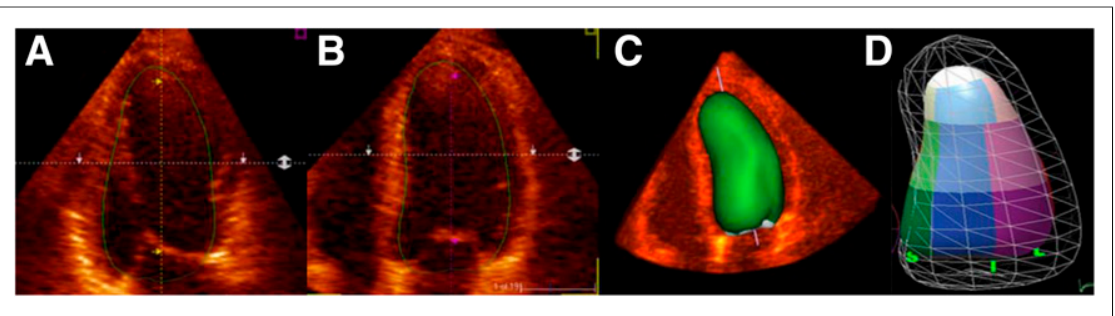

FIGURE 2. Use of $3 D$ echocardiography allows LV assumptions to be discarded. Positions of LV contours can be confirmed from 2D images (A and B) to produce 3D volume (C), and segmental evaluation can be performed (D).

Changes in the ratio between peak systolic pressure and end-systolic volume with exercise or other stress have been used as a pragmatic clinical approach (14). The measurement of preload-recruitable stroke work is well established in the experimental literature (15), but clinical use is hampered by the challenges of measuring stroke work and LV volume and of altering loading without altering inotropy. Finally, the calculation of mean cardiac power (from stroke volume, mean arterial pressure, and heart rate) or peak instantaneous power (the peak product of LV outflow and pressure during systole) can be preload-adjusted by indexing to end-diastolic volume (16). Cardiac power correlates with maximal oxygen consumption and predicts prognosis, and this methodology is not as widely used as might be expected from its ability to account for preload and afterload.

Another alternative is to select parameters that are outside the ejection phase of the cardiac cycle. The most established approach is the myocardial performance index, derived from the sum of the isovolumic contraction and isovolumic relaxation times divided by the ejection time. This measurement has been validated with invasive measures of both systolic and diastolic function (17), appears to be independent of LV geometry, is easily obtainable, and is reproducible, but it is somewhat load-dependent. Another parameter that allows assessment of LV function before the left ventricle is exposed to afterload is isovolumetric acceleration (18).

\section{Size and Shape}

The degree of LV remodeling is an important prognostic factor in patients with HF with reduced EF. Although LV dimensions remain a cornerstone of the management of valvular heart disease, the contribution of ischemic heart disease to a substantial proportion of HF means that single-dimension measurements are fraught with error because of the likelihood of regional changes. LV volume measurements using 2D echocardiography are susceptible to offaxis imaging, and they frequently underestimate the true volumes. The use of ultrasound contrast facilitates the acquisition of on-axis images and enhances contrast resolution of the true endocardial border. The use of 3D echocardiography similarly reduces the amount of underestimation, analogous to the effect of contrast, but with the benefit of reliability (19). The ongoing minor underestimation of volumes obtained using MR imaging likely relates to trabecular resolution, and it is possible to program a correction in 3D software to overcome this problem (20).

Not only LV size but also LV shape is an important clue to the severity of HF. The transition from a bullet-shaped to more spheric ventricle is a simple subjective marker that may be quantified as a sphericity index. When used with $2 \mathrm{D}$ imaging, LV shape may be influenced by regional involvement, and 3D measurements are likely preferable (21).

\section{Left Atrial (LA) Volumes}

The left atrium is considered to be a marker of long-term filling pressure analogous to the role of HbA1c as a measure of average glucose level in diabetes mellitus. LA volume is predictive of events, including atrial fibrillation. In a patient with suspected $\mathrm{HF}$, the absence of LA enlargement $\left(<35 \mathrm{~mL} / \mathrm{m}^{2}\right)$ indicates either that the process is acute or that sustained elevation of filling pressure has not occurred. LA volume is a valuable prognostic marker (22). LA volume is measured using a biplane approach in the apical views; 3D measurements have also been validated (23).

LA function has recently been shown to add incremental predictive information about the development of atrial fibrillation. The conventional measurement of atrial function involves Doppler measures to assess conduit, reservoir, and contractile function (24). The assessment of LA strain is more feasible and has the ability to measure both LA compliance and LA contraction (24).

\section{Diastolic Function}

The echocardiographic evaluation of diastolic function is dependent on transmitral flow, annular tissue Doppler, LA volume, and pulmonary vein flow (25). Echocardiographic techniques for this assessment are imperfect, with the most widely used being E/ $\mathrm{e}^{\prime}$ (the ratio between early mitral inflow velocity and mitral annular early diastolic velocity). A particular limitation of this parameter is susceptibility to loading conditions (Fig. 4); other problems are mentioned in Table 2 (26). Further, categorization of diastolic dysfunction into mild (delayed relaxation), moderate (pseudonormal), and severe (restrictive) offers prognostic staging. Unfortunately, there is only limited concordance between observers in the characterization of these entities (27).

\section{Right Ventricular (RV) Function}

The presence of RV dysfunction has important prognostic implications in patients with HF. Traditionally, RV dysfunction

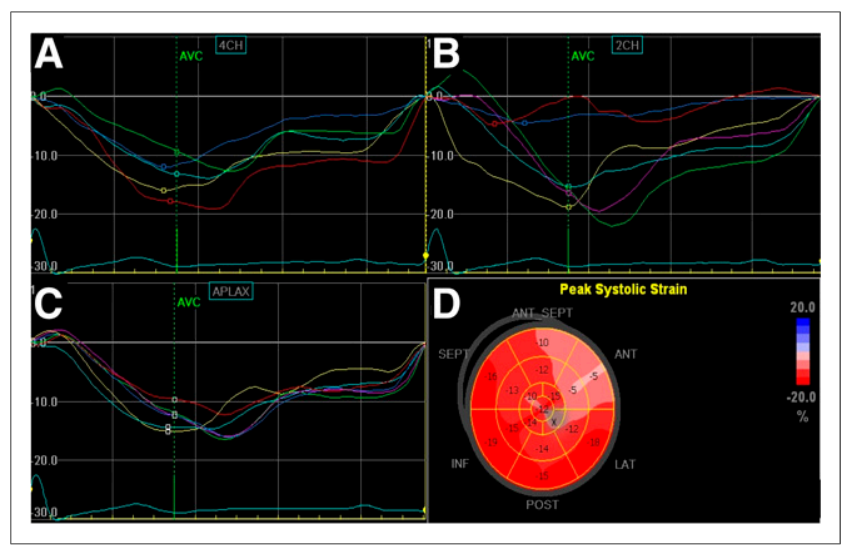

FIGURE 3. LV regional and global strain can be calculated from speckle-tracking of standard 2D images. Each LV segment is tracked throughout cycle to provide regional strain curve, and these are displayed in each apical view (4-chamber $[A], 2$-chamber $[B]$, and long-axis $[C])$. Global strain is shown in polar map display (D). In this example of patient with anterior myocardial infarction, strain curves are abnormal in apical 2-chamber view and extent of infarction is illustrated in parametric display. 


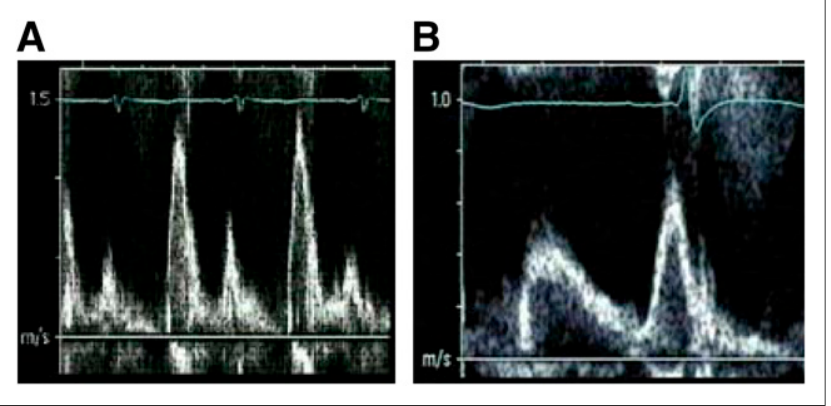

FIGURE 4. Load dependence of diastolic evaluation from transmitral flow. In this dialysis patient, imaging during fluid overload (A) showed restrictive filling pattern, reverting to delayed relaxation $(B)$ after development of euvolemia.

has been evaluated qualitatively, but the development of RV freewall strain and annular systolic tissue velocity of the RV (RF s') has been an improvement over existing quantitative measures such as tricuspid annular displacement, fractional area change, and myocardial performance index (28).

\section{Mitral Regurgitation}

The presence and severity of mitral regurgitation are important parameters that should be assessed quantitatively (29). The distinction between a failing left ventricle due to severe mitral regurgitation and severe functional mitral regurgitation due to a failing left ventricle may have important implications, and the cornerstone of this distinction is evaluation of the mitral valve. Functional mitral regurgitation is characterized by tethering of the leaflets, and a jet that is usually central or posteriorly directed in the absence of anterior leaflet prolapse. Ischemic mitral regurgitation may also be associated with a commissural jet, related to loss of support from one or the other papillary muscle (30). Even when mitral regurgitation is not severe, the spectral Doppler jet provides information about LV function; the rate of increase of velocity is analogous to the measurement of LV dP/dt (normally $>1,200 \mathrm{~mm}$ $\mathrm{Hg} / \mathrm{s}$ ), a marker of LV contractility that is especially useful in mitral regurgitation, when LV function may be overestimated by the EF.

\section{Hemodynamics}

The hemodynamic assessment of patients presenting with HF is a fundamental strength of echocardiography. The evaluation of transmitral flow provides information on LV filling pressure (1).
However, the most important aspect is probably the estimation of pulmonary artery systolic pressure, which is calculated from tricuspid regurgitation velocity and estimation of right atrial pressure from the inferior vena cava diameter in response to a sniff (24). The limitations of this approach are that it depends on a tricuspid regurgitation signal (although this is reportable in most HF patients) and carries a risk of underestimating pulmonary artery systolic pressure in the presence of severe tricuspid regurgitation, which can contravene the assumption of low right atrial pressure.

The assessment of cardiac output from volumetric approaches is based on tracing diastolic and systolic volumes or using Doppler to calculate stroke volume from blood flow, outflow tract diameter, and heart rate. The measurement of stroke volume depends on accurate estimation of LV outflow tract diameter, errors of which are squared in the course of volume calculations. Unfortunately, accurate tracing of end-diastolic and end-systolic borders is also problematic. Consequently, neither of these approaches is part of routine practice.

\section{Stress Response}

It has been widely recognized that the preservation of LV contractile reserve in response to either dobutamine or exercise stress is an important prognostic clue in both ischemic and nonischemic cardiomyopathy. More recently, RV contractile reserve has also been shown to add value (31). However, recognition of ischemia in the dilated, failing heart can be difficult, and segmental evaluation of viability with stress echocardiography even more so.

\section{Linking the Investigation to the Question}

The guidelines emphasize that no single test satisfies all imaging requirements in HF. Other modalities, discussed elsewhere in this issue of The Journal of Nuclear Medicine, can provide additional information on specific questions (especially tissue characterization). These other tests are best selected according to the imaging requirements of various indications; an approach to this process is summarized in Table 3 .

\section{SYMPTOMATIC HF}

\section{Echocardiography and Other Investigations}

The initial diagnosis of HF in many patients follows an acute presentation with pulmonary edema. Although HF is a clinical diagnosis, the symptom of dyspnea is nonspecific, particularly in the context of chronic lung disease. In this situation, echocardiography or serum levels of type B natriuretic peptide

TABLE 2

Situations in Which Use of E/e' May Be Unreliable

\begin{tabular}{ll}
\hline \multicolumn{1}{c}{ Situation } & Cause \\
\hline Fusion of E and A waves & Tachycardia \\
Unreliable measurement of E velocity & Significant mitral regurgitation $(>2+)$ \\
& Significant mitral stenosis \\
Unreliable measurement of $e^{\prime}$ velocity & Significant aortic regurgitation $(>2+)$ \\
& Severe mitral annular calcification \\
\hline & Mitral valve repair or replacement \\
& Localized wall motion abnormalities \\
\hline
\end{tabular}


TABLE 3

Selection of the Right Tool for the Job: Imaging Characteristics of Various Tests

\begin{tabular}{lll}
\hline \multicolumn{1}{c}{ Application } & Technical requirement & Technique \\
\hline LV hypertrophy, infiltration & High spatial resolution & Cardiac MR \\
LV synchrony & High temporal resolution & Tissue Doppler, strain \\
LV volumes & High contrast resolution & Cardiac MR, contrast echocardiography \\
Sequential follow-up & High repeatability & Cardiac MR, 3D echocardiography \\
Subclinical cardiomyopathy & Sensitivity to minor change & Strain \\
\hline
\end{tabular}

may facilitate the diagnosis (32). Patients with abnormal type B natriuretic peptide will need a follow-up echocardiogram to confirm HF and characterize its type.

Approximately $50 \%$ of patients in contemporary registries and studies of HF have preserved EF (33). This should not be perceived as HF with normal systolic function, because systolic function can indeed be abnormal in these patients, with the apparent paradox being explained by the insensitivity of EF to minor impairment of systolic function. Although HF with preserved EF has historically been a diagnosis of exclusion, the increasing sophistication in the measurement of myocardial deformation and diastolic function with echocardiography has led to the incorporation of these parameters in the assessment of these patients. Three aspects are of value: left atrial volume, E/e', and diastolic stage. In particular, the estimation of LV filling pressure may be helpful for guiding diuretic therapy.

\section{Etiology}

HF is a syndrome rather than a diagnosis, and the etiology of HF may be apparent from echocardiography. The first step is evaluation of the left ventricle on the basis of relative wall thickness and LV mass; morphology is categorized as normal, concentric remodeling, concentric hypertrophy, and eccentric hypertrophy (34). The first three of these morphologies may be seen with $\mathrm{HF}$ with preserved EF, whereas the fourth is characteristic of $\mathrm{HF}$ with reduced EF. Regional wall motion abnormalities and especially LV thickening suggest coronary artery disease, although myopathic processes may also show regional variation in LV function. LV hypertrophy suggests that the etiology of HF may be related to hypertension or diabetes mellitus, but asymmetric hypertrophy is consistent with a cardiomyopathy (2). In patients in whom the degree of wall thickening is not matched by the QRS magnitude, amyloid heart disease should be considered, particularly when tissue velocity or strain is low. A particular strain pattern of apical sparing has high specificity for amyloidosis (35) but probably modest sensitivity. In some situations, reevaluation of the LV response with stress may facilitate the diagnosis of coronary artery disease. This approach may be particularly important in patients with suspected HF with preserved EF, in whom dyspnea may be an angina equivalent that simply reflects the presence of coronary disease.

\section{Differential Diagnosis and Complications}

Echocardiography also plays a vital role in the recognition of other disease entities when HF symptoms are not attributable to myocardial dysfunction. The most important groups of patients are those with valvular heart disease and those with pericardial disease. Constrictive pericarditis should always be considered as a differential diagnosis in patients with HF with preserved EF, especially those with a history of previous cardiac surgery.

Patients with severe LV dysfunction are at risk for the development of LV thrombus. This risk may not be realized on standard 2D echocardiography, and there should be a low threshold for the use of contrast echocardiography in settings such as ischemic LV dysfunction with an apical aneurysm or HF patients presenting with a transient ischemic attack or stroke (36). Unstable HF patients may have pericardial effusions, which are readily recognizable with handheld echocardiography.

TABLE 4

Markers of Response in Patients Undergoing Cardiac Resynchronization Therapy

\begin{tabular}{|c|c|c|}
\hline Parameter & Measure & Response at $6 \mathrm{mo}$ \\
\hline \multirow[t]{3}{*}{ Clinical } & Composite & "Improved" \\
\hline & New York Heart Association class & Decrease by at least one class \\
\hline & $\begin{array}{l}\text { Minnesota Living with Heart Failure } \\
\text { questionnaire }\end{array}$ & $\geq 9$-point decrease \\
\hline $\begin{array}{l}\text { Exercise } \\
\text { capacity }\end{array}$ & 6-min walk & $\begin{array}{l}\geq 10 \% \text { improvement (or any improvement if walked zero at } \\
\text { baseline) }\end{array}$ \\
\hline \multirow[t]{4}{*}{ LV features } & LV volume & $\geq 15 \%$ decrease \\
\hline & LV EF & $\geq 5 \%$ increase \\
\hline & LV mass & Any decrease \\
\hline & Tei index & Any decrease \\
\hline Mitral valve & Mitral regurgitation & Decrease in mitral regurgitation severity \\
\hline
\end{tabular}


TABLE 5

Differences Between Echocardiograms According to Parameter

\begin{tabular}{lcc}
\hline \multicolumn{1}{c}{ Parameter } & Absolute difference & Relative difference \\
\hline$\Delta$ Left ventricular EF & $8.1 \% \pm 11.5 \%$ & $17 \% \pm 30 \%$ \\
$\Delta$ Left atrial area & $4.0 \pm 5.2 \mathrm{~cm}^{2}$ & $17 \% \pm 23 \%$ \\
$\Delta$ Tissue Em & $2.1 \pm 2.7 \mathrm{~cm} / \mathrm{s}$ & $27 \% \pm 36 \%$ \\
$\Delta \mathrm{E} / \mathrm{e}^{\prime}$ & $5.0 \pm 7.0$ & $46 \% \pm 64 \%$ \\
& & \\
\hline Em $=$ mitral annular tissue diastolic velocity. & \\
\hline
\end{tabular}

\section{ASSESSMENT OF PROGNOSIS}

HF is widely recognized as a highly morbid disease, but morbidity and mortality rates are not uniform. Echocardiography is an important component of risk evaluation; mortality is predicted by the severity of LV remodeling, EF, strain (especially in HF with preserved EF), diastolic dysfunction and LV filling pressure, RV function, pulmonary hypertension, and mitral regurgitation (37).

The prognostic assessment of HF patients relates not only to mortality but also to risk of hospital readmission (38). There is particular interest in this endpoint since its selection by the Centers for Medicare and Medicaid Services as a marker of quality, with consequent financial incentives. Generally, EF has not been a reliable marker of readmission risk-perhaps reflecting the high frequency of $\mathrm{HF}$ with preserved EF. In contrast, LV strain has been shown to predict $\mathrm{HF}$ admission (39). Perhaps more important is the protection of HF readmission based on hemodynamic assessment, including LV filling pressure, pulmonary hypertension, and right atrial pressure (40).

\section{SELECTION OF PATIENTS FOR DEVICE THERAPY}

Selection of patients for a wide variety of device therapies is also reliant on echocardiographic assessment. In patients with LV dysfunction, these decisions relate to implantable cardioverter defibrillators and cardiac resynchronization therapy, whereas in advanced HF, echocardiography may contribute to evaluation for $\mathrm{LV}$ and RV assistive devices.

\section{Implantable Cardioverter Defibrillators}

The selection of patients for implantable cardioverter defibrillator therapy depends on the evaluation of EF, although the obsessive use of individual cutoffs in the setting seems imprudent. Neither the modality of EF nor the use of a core laboratory was stipulated in most of the trials that supported the incorporation of device therapy into guidelines (41). Moreover, although it is true that the risk of sudden cardiac death is proportionate to the severity of LV dysfunction, the much larger number of at-risk patients with preserved EF led this preserved EF group to account for greater numbers of deaths despite the fact that the individual risk of death is lower. A promising alternative strategy to understanding the risk of arrhythmia and sudden cardiac death has been the evaluation of myocardial fibrosis. It appears that heterogeneous scarring is an important driver of arrhythmia, and it appears that temporal dispersion of myocardial contraction-which can be measured by strain-parallels the magnitude of this problem $(42,43)$.

\section{Cardiac Resynchronization Therapy}

Although the use of cardiac resynchronization therapy has been justified by prognostic benefit across the population of HF patients and the wide QRS (44), it is also known that symptomatic or functional benefit is not uniform in wide QRS patients. Unfortunately, defining the response to cardiac resynchronization therapy is difficult, with several potential arbiters of these findings (Table 4), and the use of this concept of nonresponsiveness has been highly controversial (45).

Given the prognostic benefit of this intervention, any test to predict nonresponsiveness would have to be highly specific in order to justify failure to administer a potentially life-saving therapy. Nonetheless, it is often not recognized that the ventricular mechanics, and sometimes symptoms, may sometimes worsen if mechanical dyssynchrony is ignored. Although several echocardiographic measurements, particularly involving tissue Doppler, have proven difficult outside major centers, the use of myocardial strain to document the site of maximal delay has now been shown in more than one randomized trial to benefit the outcome of cardiac resynchronization therapy $(46,47)$. In addition, echocardiographic assessment of candidates for cardiac resynchronization therapy can predict nonresponse on the basis of nonsynchrony markers, including the severity of ventricular enlargement, RV involvement, and hemodynamic evaluation (48).

\section{Assistive Devices}

The selection and monitoring of patients for assistive devices is a highly specialized topic (49). One of the most important aspects of the evaluation before implantation relates to the assessment of $\mathrm{RV}$ function (50). Individuals with severe RV dysfunction may require a biventricular assistive device and recognition of this before device implantation is important. However, although the measurement of RV strain seems to be a worthwhile parameter when this decision is to be made, assessment of RV strain is complicated and cannot be simplified to pertain to a single parameter.

\section{REASSESSMENT}

Patients with HF are often reassessed using imaging, and the appropriateness of this strategy is controversial (51). In symptomatic

TABLE 6

Heart Failure Stages as Defined by Classification System of American College of Cardiology and American Heart Association

\begin{tabular}{ccccc}
\hline Stage & Risk factors for HF & Structural/functional heart disease & History of HF & Clinical congestive HF \\
\hline Normal & No & No & No & - \\
Stage A & Yes & No & No & No \\
Stage B & - & Yes & No & No \\
Stage C1 & - & Yes & Yes & - \\
Stage C2 & - & Yes & Yes & Yes \\
Stage D & - & Yes & & \\
\hline
\end{tabular}


TABLE 7

Strengths and Weaknesses of Different Imaging Modalities

\begin{tabular}{|c|c|c|c|c|c|c|c|}
\hline Modality & Accessibility & Patient-friendliness & Preclinical HF & Accuracy & Hemodynamics & Regional function & Reproducibility \\
\hline Echocardiography & ++ & ++ & ++ & + & ++ & + & + \\
\hline Nuclear & + & + & - & + & $+/-$ & $+/-$ & + \\
\hline $\mathrm{CT}$ & + & + & - & + & - & + & + \\
\hline Cardiac MR & $+/-$ & + & ++ & ++ & + & ++ & ++ \\
\hline
\end{tabular}

patients, including those readmitted to the hospital without a ready explanation such as medication nonadherence, repeated echocardiography is deemed appropriate. In stable individuals, repeated echocardiography is considered rarely appropriate. In some situations, such as stable patients who have not attained the EF threshold for device therapy, follow-up imaging may be considered, but the justifiable frequency of reassessment for this cause is unclear.

The fundamental requirement for repeated imaging is reliabilityknowing that EF correlates with the results of another test is relevant but less important than knowing that observed changes are actual changes rather than a problem with testing reproducibility. In patients undergoing repeated imaging, the hemodynamic components of the echocardiographic evaluation (right atrial pressure, pulmonary hypertension, LV filling pressure, and stroke volume) are probably the most important, but some are highly variable (Table 5). In particular, the serial assessment of EF with 2D echocardiography should be used with great caution. Because of the difficulty in replicating the same views, the $95 \%$ confidence intervals for repeated $2 \mathrm{D} \mathrm{EF}$ are on the order of $10 \%$ (9). The superiority of 3D echocardiography over 2D echocardiography is most valuable in the assessment of LV volumes, with a similar accuracy for EF, but this observation hides the substantial benefit of 3D echocardiography in terms of reproducibility (52). Myocardial strain may also be an effective means of repeated assessment.

\section{EARLY DIAGNOSIS OF HF}

The adverse outcomes of clinical HF have spurred efforts to prevent progression of LV dysfunction to this stage (Table 6). In recognition of the patient's progression through the natural history of HF, the American College of Cardiology and American Heart Association (53) have described a series of stages based on cardiac risk factors, evidence of cardiac involvement, clinical manifestations, and refractoriness to treatment (Table 6). The distinction between stage A (HF risk factors including hypertension, type 2 diabetes mellitus, and obesity) and stage B (structural abnormalities such as LV hypertrophy and evidence of past infarction) has important implications for medical management and prognosis.

Because HF risk factors are highly prevalent in the elderly (54), the recognition of stage $\mathrm{B} \mathrm{HF}$ is a population health challenge, and echocardiography - an inexpensive, widely available, safe, and noninvasive technique-is attractive for this purpose. The LV structural and functional remodeling associated with early stages of ischemic HF is currently easier to recognize than nonischemic etiologies, because mild LV hypertrophy is difficult to recognize. Because the population-attributable risk of nonischemic etiologies (especially diabetes mellitus) is becoming more important, recent work on the use of diastolic evaluation and myocardial mechanics may become more important as a diagnostic approach to this problem (55).

\section{CONCLUSION}

Cardiac imaging is indispensable in the management of HF. Echocardiography is the most widely used test for this purpose and the most versatile in terms of providing clinically relevant information (Table 7). In addition to providing information on EF, echocardiography provides information on LV volume, diastolic function, RV function, hemodynamics, and valvular regurgitation that has important prognostic and therapeutic implications. However, although analogous measurements are obtainable from multiple modalities, the data they provide are often discordant, especially LV volumes. These inconsistencies arise from inherent differences in the temporal, contrast, and spatial resolution of the techniques, and extreme caution should be applied in the comparison of measurements between modalities.

Echocardiography is important at every stage of HF, but recent developments in the assessment of LV strain are especially important to the recognition of the preclinical phases of nonischemic HF. Ongoing research is needed to refine applications in this setting, especially by assembling evidence of prognostic and therapeutic implications.

\section{DISCLOSURE}

No potential conflict of interest relevant to this article was reported.

\section{REFERENCES}

1. Kirkpatrick JN, Vannan MA, Narula J, Lang RM. Echocardiography in heart failure: applications, utility, and new horizons. J Am Coll Cardiol. 2007;50:381-396.

2. Arbustini E, Narula N, Dec GW, et al. The MOGE(S) classification for a phenotypegenotype nomenclature of cardiomyopathy: endorsed by the World Heart Federation. J Am Coll Cardiol. 2013;62:2046-2072.

3. Marwick TH. Methods used for the assessment of LV systolic function: common currency or tower of Babel? Heart. 2013;99:1078-1086.

4. Abraham T, Kass D, Tonti G, et al. Imaging cardiac resynchronization therapy. JACC Cardiovasc Imaging. 2009;2:486-497.

5. Dalen H, Gundersen GH, Skjetne K, et al. Feasibility and reliability of pocketsize ultrasound examinations of the pleural cavities and vena cava inferior performed by nurses in an outpatient heart failure clinic. Eur J Cardiovasc Nurs. August 13, 2014 [Epub ahead of print].

6. Foster E, Cahalan MK. The search for intelligent quantitation in echocardiography: "eyeball," "trackball" and beyond. J Am Coll Cardiol. 1993;22:848-850.

7. Lang RM, Bierig M, Devereux RB, et al. Recommendations for chamber quantification. J Am Soc Echocardiogr. 2005;18:1440-1463.

8. Dorosz JL, Lezotte DC, Weitzenkamp DA, Allen LA, Salcedo EE. Performance of 3-dimensional echocardiography in measuring left ventricular volumes and ejection fraction: a systematic review and meta-analysis. J Am Coll Cardiol. 2012;59:1799-1808.

9. Thavendiranathan P, Grant AD, Negishi T, et al. Reproducibility of echocardiographic techniques for sequential assessment of left ventricular ejection fraction 
and volumes: application to patients undergoing cancer chemotherapy. J Am Coll Cardiol. 2013;61:77-84.

10. Senni M, Rodeheffer RJ, Tribouilloy CM, et al. Use of echocardiography in the management of congestive heart failure in the community. J Am Coll Cardiol. 1999;33:164-170.

11. Tribouilloy C, Rusinaru D, Mahjoub H, et al. Impact of echocardiography in patients hospitalized for heart failure: a prospective observational study. Arch Cardiovasc Dis. 2008;101:465-473.

12. Mor-Avi V, Lang RM, Badano LP, et al. Current and evolving echocardiographic techniques for the quantitative evaluation of cardiac mechanics. J Am Soc Echocardiogr. 2011;24:277-313.

13. Reichek N, Wilson J, St John SM, et al. Noninvasive determination of left ventricular end-systolic stress: validation of the method and initial application. Circulation. 1982;65:99-108.

14. Jellis CL, Jenkins C, Leano R, Martin JH, Marwick TH. Reduced pressurevolume response to exercise: a marker of subclinical myocardial disease in type 2 diabetes. Circ Cardiovasc Imaging. 2010;3:443-449.

15. Glower DD, Spratt JA, Snow ND, et al. Linearity of the Frank-Starling relationship in the intact heart: the concept of preload recruitable stroke work. Circulation. 1985;71:994-1009.

16. Mandarino WA, Pinsky MR, Gorcsan J III. Assessment of left ventricular contractile state by preload-adjusted maximal power using echocardiographic automated border detection. J Am Coll Cardiol. 1998;31:861-868.

17. Tei C, Nishimura RA, Seward JB, Tajik AJ. Noninvasive Doppler-derived myocardial performance index: correlation with simultaneous measurements of cardiac catheterization measurements. J Am Soc Echocardiogr. 1997;10:169-178.

18. Vogel M, Cheung MM, Li J, et al. Noninvasive assessment of left ventricular force-frequency relationships using tissue Doppler-derived isovolumic acceleration: validation in an animal model. Circulation. 2003;107:1647-1652.

19. Jenkins C, Moir WS, Chan J, Marwick TH. Left ventricular volume measurement with echocardiography: a comparison of left ventricular opacification, 3D echocardiography or both with magnetic resonance imaging. Eur Heart J. 2009;30:98-106.

20. Thavendiranathan P, Liu S, Verhaert D, et al. Feasibility, accuracy, and reproducibility of real-time full-volume 3D transthoracic echocardiography to measure LV volumes and systolic function: a fully automated endocardial contouring algorithm in sinus rhythm and atrial fibrillation. JACC Cardiovasc Imaging. 2012;5:239-251.

21. Mannaerts HF, van der Heide JA, Kamp O, et al. Early identification of left ventricular remodelling after myocardial infarction, assessed by transthoracic 3D echocardiography. Eur Heart J. 2004;25:680-687.

22. Tsang TS, Barnes ME, Bailey KR, et al. Left atrial volume: important risk marker of incident atrial fibrillation in 1655 older men and women. Mayo Clin Proc. 2001;76:467-475.

23. Mor-Avi V, Jenkins C, Kuhl HP, et al. Real-time 3D echocardiographic quantification of left atrial volume: multicenter study for validation with magnetic resonance imaging. JACC Cardiovasc Imaging. 2012;5:769-777.

24. To AC, Flamm SD, Marwick TH, Klein AL. Clinical utility of multimodality LA imaging: assessment of size, function, and structure. JACC Cardiovasc Imaging. 2011;4:788-798.

25. Nagueh SF, Appleton CP, Gillebert TC, et al. Recommendations for the evaluation of left ventricular diastolic function by echocardiography. J Am Soc Echocardiogr. 2009;22:107-133.

26. Park JH, Marwick TH. Use and limitations of E/e' to assess ventricular filling pressure by echocardiography. J Cardiovasc Ultrasound. 2011;19:169-173.

27. Unzek S, Popovic ZB, Marwick TH. Effect of recommendations on inter-observer consistency of diastolic function evaluation: an international multicenter study. JACC Cardiovasc Imaging. 2011;4:460-467.

28. Rudski LG, Lai WW, Afilalo, J et al. Guidelines for the echocardiographic assessment of the right heart in adults. J Am Soc Echocardiogr. 2010;23:685-713.

29. Zoghbi WA, Enriquez-Sarano M, Foster E, et al. Recommendations for evaluation of the severity of native valvular regurgitation with two-dimensional and Doppler echocardiography. J Am Soc Echocardiogr. 2003;16:777-802.

30. Dal-Bianco JP, Beaudoin J, Handschumacher MD, Levine RA. Basic mechanisms of mitral regurgitation. Can J Cardiol. 2014;30:971-981.

31. Matsumoto K, Tanaka H, Onishi A. Bi-ventricular contractile reserve offers an incremental prognostic value for patients with dilated cardiomyopathy. Eur Heart J Cardiovasc Imaging. April 6, 2015 [Epub ahead of print].

32. Kotecha T, Fox K. Investigating suspected heart failure [comment]. BMJ. 2013;346:f2442.

33. Bhatia RS, Tu JV, Lee DS, et al. Outcome of heart failure with preserved ejection fraction in a population-based study. $N$ Engl J Med. 2006;355:260-269.
34. Marwick TH, Gillebert T, Aurigemma G, et al. Recommendations on the use of echocardiography in adult hypertension. Eur Heart J Cardiovasc Imaging. In press.

35. Phelan D, Collier P, Plana JC, et al. Relative "apical sparing" of longitudinal strain using 2-dimensional speckle-tracking echocardiography is both sensitive and specific for the diagnosis of cardiac amyloidosis. Heart. 2012;98:1442-1448.

36. Mulvagh SL, Rakowski H, Vannan MA, et al. American Society of Echocardiography consensus statement on the clinical applications of ultrasonic contrast agents in echocardiography. J Am Soc Echocardiogr. 2008;21:1179-1201.

37. Agha SA, Kalogeropoulos AP, Shih J, et al. Echocardiography and risk prediction in advanced heart failure: incremental value over clinical markers. $J$ Card Fail. 2009;15:586-592.

38. Chen J, Normand SL, Wang Y, Krumholz HM. National and regional trends in heart failure hospitalization and mortality rates for Medicare beneficiaries, 19982008. JAMA. 2011;306:1669-1678.

39. Saito M, Negishi K, Eskandari M, et al. Association of left ventricular strain with 30-day mortality and readmission in patients with heart failure. J Am Soc Echocardiogr. March 14, 2015 [Epub ahead of print].

40. Thavendiranathan P, Yingchoncharoen T, Grant A, et al. Prediction of 30-day heart failure-specific readmission risk by echocardiographic parameters. Am J Cardiol. 2014;113:335-341.

41. Bardy GH, Lee KL, Mark DB, et al. Amiodarone or an implantable cardioverterdefibrillator for congestive heart failure. N Engl J Med. 2005;352:225-237.

42. Haugaa $\mathrm{KH}$, Grenne BL, Eek $\mathrm{CH}$, et al. Strain echocardiography improves risk prediction of ventricular arrhythmias after myocardial infarction. JACC Cardiovasc Imaging. 2013;6:841-850.

43. Haugaa KH, Goebel B, Dahlslett T, et al. Risk assessment of ventricular arrhythmias in patients with nonischemic dilated cardiomyopathy by strain echocardiography. J Am Soc Echocardiogr. 2012;25:667-673.

44. Vardas PE, Auricchio A, Blanc JJ, et al. Guidelines for cardiac pacing and cardiac resynchronization therapy: the task force for cardiac pacing and cardiac resynchronization therapy of the European Society of Cardiology—developed in collaboration with the European Heart Rhythm Association. Eur Heart J. 2007;28:2256-2295.

45. Francis DP. How easily can omission of patients, or selection amongst poorlyreproducible measurements, create artificial correlations? Methods for detection and implications for observational research design in cardiology. Int $J$ Cardiol. 2013;167:102-113.

46. Khan FZ, Virdee MS, Palmer CR, et al. Targeted left ventricular lead placement to guide cardiac resynchronization therapy: the TARGET study-a randomized, controlled trial. J Am Coll Cardiol. 2012;59:1509-1518.

47. Saba S, Marek J, Schwartzman D, et al. Echocardiography-guided left ventricular lead placement for cardiac resynchronization therapy: results of the Speckle Tracking Assisted Resynchronization Therapy for Electrode Region trial. Circ Heart Fail. 2013;6:427-434.

48. Park JH, Negishi K, Grimm R, et al. Echocardiographic predictors of reverse remodeling after cardiac resynchronization therapy and subsequent events. Circ Cardiovasc Imaging. 2013;6:864-872.

49. Todaro MC, Khandheria BK, Paterick TE, Umland MM, Thohan V. The practical role of echocardiography in selection, implantation, and management of patients requiring LVAD therapy. Curr Cardiol Rep. 2014;16:468.

50. Grant AD, Smedira N, Starling R, Marwick TH. Independent and incremental role of quantitative right ventricular evaluation for prediction of right ventricular failure after left ventricular assist device implantation. J Am Coll Cardiol. 2012;60:521-528.

51. Douglas PS, Garcia MJ, Haines DE, et al. ACCF/ASE/AHA/ASNC/HFSA/HRS/ SCAI/SCCM/SCCT/SCMR 2011 appropriate use criteria for echocardiography. J Am Coll Cardiol. 2011;57:1126-1166.

52. Jenkins C, Bricknell K, Hanekom L, Marwick TH. Reproducibility and accuracy of echocardiographic measurements of left ventricular parameters using realtime 3D echocardiography. J Am Coll Cardiol. 2004;44:878-886.

53. Yancy CW, Jessup M, Bozkurt B, et al. 2013 ACCF/AHA guideline for the management of heart failure: a report of the American College of Cardiology Foundation/American Heart Association Task Force on Practice Guidelines. $J$ Am Coll Cardiol. 2013;62:e147-e239.

54. Ammar KA, Jacobsen SJ, Mahoney DW, et al. Prevalence and prognostic significance of heart failure stages: application of the American College of Cardiology/ American Heart Association heart failure staging criteria in the community. Circulation. 2007;115:1563-1570.

55. Kosmala W, Jellis C, Marwick TH. Exercise limitation associated with asymptomatic left ventricular impairment: analogy with stage B heart failure. J Am Coll Cardiol. 2015;65:257-266. 